\title{
STUDY OF A STATIC AND VERTICALLY FREE-TO-OSCILLATE 4:1 RECTANGULAR CYLINDER BY MEANS OF 2D URANS SIMULATIONS
}

\author{
ANTONIO J. ÁLVAREZ, FÉLIX NIETO \& SANTIAGO HERNÁNDEZ \\ University of La Coruña, Spain
}

\begin{abstract}
Flexible structures, such as cable-supported bridges, are prone to suffer from vortex-induced vibrations (VIV) under wind flow, as their span lengths are steadily growing in the last decades. VIV is a phenomenon that takes place at reduced wind speeds. The movements of the structure at VIV are selflimited and their frequency corresponds with the natural frequency of the structure (lock in). Therefore, VIV affects the structure's serviceability and can cause fatigue related damage. Hence, the need for identifying and avoiding this phenomenon at the early design stages is a key issue in long-span bridges design. In the present study a rectangular cylinder of width to depth ratio $4: 1$, which is a common simplification of a bridge deck cross section, is analysed for the static case as well as undergoing free vibration in the vertical direction under wind flow. These analyses have been carried out by 2D URANS CFD simulations, adopting two different turbulence models: the $k-\omega$ SST, which is based upon the Boussinesq eddy-viscosity approximation, and the Reynolds Stress Model, which directly calculates the components of the specific Reynolds stresses. For the static case the force coefficients, Strouhal number and the pressure coefficient distributions were calculated and compared with the available experimental data. In the case of the free-to-oscillate 4:1 rectangular cylinder, the oscillation amplitudes are compared with wind tunnel data reported in the literature. In addition, the frequencies and phase-lags between the time-dependent lift coefficient and the vertical oscillations are studied.

Keywords: 4:1 rectangular cylinder, vortex shedding, vortex-induced vibrations, force coefficients, URANS, Reynolds Stress Model, CFD, pressure distribution.
\end{abstract}

\section{INTRODUCTION}

The use of rectangular cylinders, as the cross-section of structural members is widely spread. Moreover, rectangular cylinders of width to depth ratios 4:1 and 5:1 are often considered to be a generic simplified bridge deck cross section. These geometries present fixed flow separation points and may show large amplitudes of oscillation at lock-in during VIV [1].

Rectangular cylinders of different width to depth ratios have been studied by means of experimental campaigns for the static, the forced-oscillation and free-to-oscillate cases for many years. For the case of static cylinders with ratios ranging from 0.1 to 4.0, Nakaguchi et al. [2] studied their pressure coefficient distributions, drag coefficient and Strouhal number. The unsteady flow around static rectangular cylinders with ratios 0.4, 1.0, 2.0 and 4.0 was studied by Mizota and Okajima [3]. For rectangular cylinders with side ratios ranging from 0.8 to 6 Okajima [4] included in his studies velocity and Strouhal number measurements.

Moving to the dynamic response problem, Washizu et al. [5], Washizu et al. [6] studied the aeroelastic responses of rectangular cylinders of ratios ranging from 1 to 4 in heaving and pitch oscillation. Apart from studying the VIV of rectangular cylinders with ratios 2, 3, 4 and 5; Komatsu and Kobayashi [7] studied the VIV of other geometries, and some flow features for these cases undergoing vertical forced oscillation. Recently, Marra et al. [1], Marra et al. [8] proposed an empirical formulation relating the damping of the vertically free to oscillate configuration of a 4:1 rectangular cylinder with the maximum VIV amplitude, after having carried out an extensive experimental campaign. 
The numerical approach has been also considered for the study of the aerodynamic response of rectangular cylinders. In that sense, Ohya et al. [9] studied the vortex shedding over rectangular cylinders with ratios ranging from 3 to 9 . Mainly in the frame of the BARC initiative, the ratio 5 rectangular cylinder has been thoroughly studied, both numerically and experimentally, in the recent years [10]-[12]. For the ratio 2 and 4 rectangular cylinders, Shimada and Ishihara [13] studied the VIV using a modified $k-\epsilon$ model. Very little, apart from the last reference, about vertically free to oscillate response of rectangular cylinders by means 2D URANS simulations can be found. In that sense, the present study tries to assess the feasibility of these models for the identification of the VIV phenomenon, getting advantage of the relatively moderate computational cost associated with this type of approach when compared with the computationally more expensive 3D LES simulations [14], Nguyen et al. [15].

\subsection{Governing equations}

\section{FORMULATION}

The flow around the 4:1 rectangular cylinder considered in this work is modelled by the Navier-Stokes equations, whose time averaging in conservative form yields the Unsteady Reynolds-Averaged Navier-Stokes (URANS) equations [16]:

$$
\begin{gathered}
\frac{\partial U_{i}}{\partial x_{i}}=0 \\
\rho \frac{\partial U_{i}}{\partial t}+\rho U_{j} \frac{\partial U_{i}}{\partial x_{j}}=-\frac{\partial P}{\partial x_{i}}+\frac{\partial}{\partial x_{j}}\left(2 \mu S_{i j}-\rho \overline{u_{i}^{\prime} u_{j}^{\prime}}\right)
\end{gathered}
$$

where $U_{i}$ is the mean velocity vector, $x_{i}$ is the position vector, $\rho$ is the fluid density, $t$ is the time, $P$ is the mean pressure, $\mu$ is the fluid viscosity, $S_{i j}$ is the mean strain-rate tensor and $u_{i}^{\prime}$ is the fluctuating velocity.

The term $-\overline{u_{i}^{\prime} u_{j}^{\prime}}$ is the so-called specific Reynolds stress tensor $\left(\tau_{i j}\right)$. At this point it is necessary to differentiate between the models which rely on the Boussinesq assumption in order to model the Reynolds stresses or attempt to calculate them directly. The $k-\omega$ SST model is allocated in the former category, meanwhile the Reynolds Stress Transport Model (RSTM) falls into the latter one, which are also known as second-moment closure models.

The $k-\omega$ SST model uses the Boussinesq assumption for calculating the Reynolds stress tensor. This assumption is expressed as:

$$
\tau_{i j}=2 \nu_{T} S_{i j}-\frac{2}{3} k \delta_{i j}
$$

where $\nu_{T}$ is the kinematic eddy viscosity and $k$ is the kinetic energy per unit mass of the turbulent fluctuation.

Eqns (4) to (6) show the transport equations for the turbulent kinetic energy $(k)$, the specific dissipation rate $(\omega)$ and auxiliary relationships according to Menter and Esch [17]

$$
\begin{gathered}
\rho \frac{\partial k}{\partial t}+\rho \frac{\partial U_{j} k}{\partial x_{j}}=\widetilde{P_{k}}-\beta^{*} \rho \omega k+\frac{\partial}{\partial x_{j}}\left(\Gamma_{k} \frac{\partial k}{\partial x_{j}}\right) \\
\rho \frac{\partial \omega}{\partial t}+\rho \frac{\partial U_{j} \omega}{\partial x_{j}}=\frac{\gamma}{\nu_{t}} P_{k}-\beta \rho \omega^{2}+\frac{\partial}{\partial x_{j}}\left(\Gamma_{\omega} \frac{\partial \omega}{\partial x_{j}}\right)+\left(1-F_{1}\right) 2 \rho \sigma_{w 2} \frac{1}{\omega} \frac{\partial k}{\partial x_{j}} \frac{\partial \omega}{\partial x_{j}}
\end{gathered}
$$




$$
\begin{gathered}
\Gamma_{k}=\mu+\frac{\mu_{t}}{\sigma_{k}}, \Gamma_{\omega}=\mu+\frac{\mu_{t}}{\sigma_{\omega}}, P_{k}=\tau_{i j} \frac{\partial U_{i}}{\partial x_{j}}, \\
\widetilde{P_{k}}=\min \left(P_{k} ; c_{1} \epsilon\right), \nu_{t}=\rho \frac{a_{1} k}{\max \left(a_{1} \omega ; S \cdot F_{2}\right)}, S=\sqrt{2 S_{i j} S_{i j}},
\end{gathered}
$$

where $\mu$ is the dynamic viscosity, $\mu_{t}$ is the turbulent dynamic viscosity, $\beta, \beta^{*}, \gamma, \sigma_{\omega}, \sigma_{\omega 2}$, $\sigma_{k}, a_{1}$ and $c_{1}$ are model constants, finally $F_{1}$ and $F_{2}$ are auxiliary functions.

Moving into the RSTM, eqn (7) shows the formulation of the Reynolds stresses transport equation [16]

$$
\begin{aligned}
& \frac{\partial \tau_{i j}}{\partial t}=-\left(U_{k} \frac{\partial \tau_{i j}}{\partial x_{k}}+\tau_{i k} \frac{\partial U_{j}}{\partial x_{k}}+\tau_{j k} \frac{\partial U_{i}}{\partial x_{k}}\right)+\nu \frac{\partial^{2} \tau_{i j}}{\partial x_{k}^{2}} \\
& +2 \nu \overline{\frac{\partial u_{i}^{\prime}}{\partial x_{k}} \frac{\partial u_{j}^{\prime}}{\partial x_{k}}}+\overline{\frac{p^{\prime}}{\rho}\left(\frac{\partial u_{i}^{\prime}}{\partial x_{j}}+\frac{\partial u_{j}^{\prime}}{\partial x_{i}}\right)}+\frac{\partial}{\partial x_{k}}\left(\overline{u_{i}^{\prime} u_{j}^{\prime} u_{k}^{\prime}}\right) .
\end{aligned}
$$

In eqn (7) all the elements of the second line need to be modelled. The terms in that line correspond to the dissipation tensor, the pressure-strain correlation tensor and the turbulent transport tensor, where pressure fluctuations are neglected. As the Reynolds stresses naturally account for flow effects that eddy viscosity models can not (turbulence anisotropy, streamline curvature, swirl rotation and high strain rates), a priori they can predict more complex flows than the latter ones. In this application case, it has been adopted the Elliptic Blending Model [18] for modelling the pressure strain,which is the second term in the second line in eqn (7). The Elliptic Blending Model is the low-Reynolds number variant of the Quadratic Pressurestrain model, based on a inhomogeneous near-wall formulation of the quasi-linear quadratic pressure-strain term. The viscous sublayer and log-layer formulations of the pressure-strain term must be blended, requiring the solution of an elliptic equation for the blending parameter $\alpha[19]$.

\subsection{Force coefficients, Strouhal number and pressure coefficient}

The force coefficients and Strouhal number $(S t)$, are calculated as shown in the following equations. The positive directions are the upward direction for the lift and the clockwise rotation for the moment. Positive drag force is taken in the along wind direction

$$
C_{d}=\frac{F_{D}}{\frac{1}{2} \rho U^{2} D} \quad C_{l}=\frac{F_{L}}{\frac{1}{2} \rho U^{2} D} \quad C_{m}=\frac{M}{\frac{1}{2} \rho U^{2} D^{2}} \quad S t=\frac{f D}{U} .
$$

The mean pressure coefficient $\left(\overline{C_{p}}\right)$ and its standard deviation $\left(\widetilde{C_{p}}\right)$ are calculated as shown by eqn (9)

$$
\overline{C_{p}}=\frac{\bar{p}}{\frac{1}{2} \rho U^{2}} \quad \widetilde{C_{p}}=\frac{\sigma_{p}}{\frac{1}{2} \rho U^{2}}
$$

where $\bar{p}$ is the mean pressure and $\sigma_{p}$ is the pressure standard deviation.

\subsection{Numerical settings}

\section{COMPUTER MODELLING}

The Reynolds Averaged Navier-Stokes equations are solved by means of the CFD software OpenFOAM for the two-equation $k-\omega$ SST model. The diffusive terms were discretised by using the second order central difference scheme while for the convective terms the 
linear upwind differential scheme is used. Advancement in time was performed by a first order implicit scheme. Finally, the pressure-velocity coupling was solved by the PIMPLE algorithm. The maximum Courant $(\mathrm{Co})$ number was limited to 1 at every time step.

For the simulations where the RSTM is used, STAR-CCM+ has been the solver of choice. In these simulations, for the convective terms a second order scheme was used, the gradients were solved by the HybridGauss-LSQ scheme and the advancement in time was done by a second order scheme.

For the dynamic simulations, the Arbitrary Lagrangian Eulerian algorithm was applied to incorporate the movement of the mesh into the governing fluid equations. The cylinder

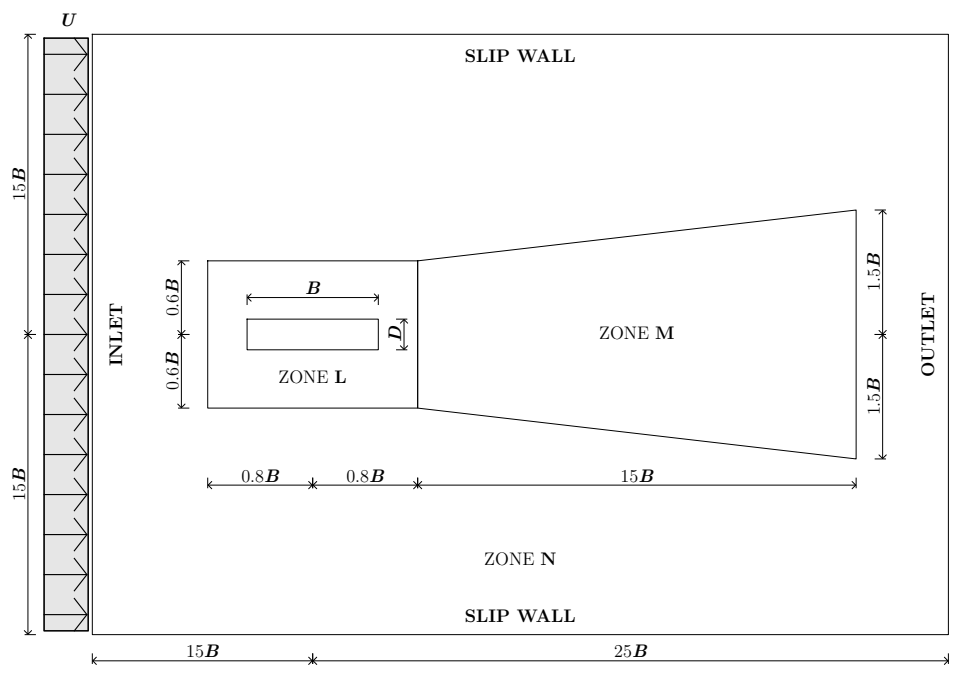

(a)

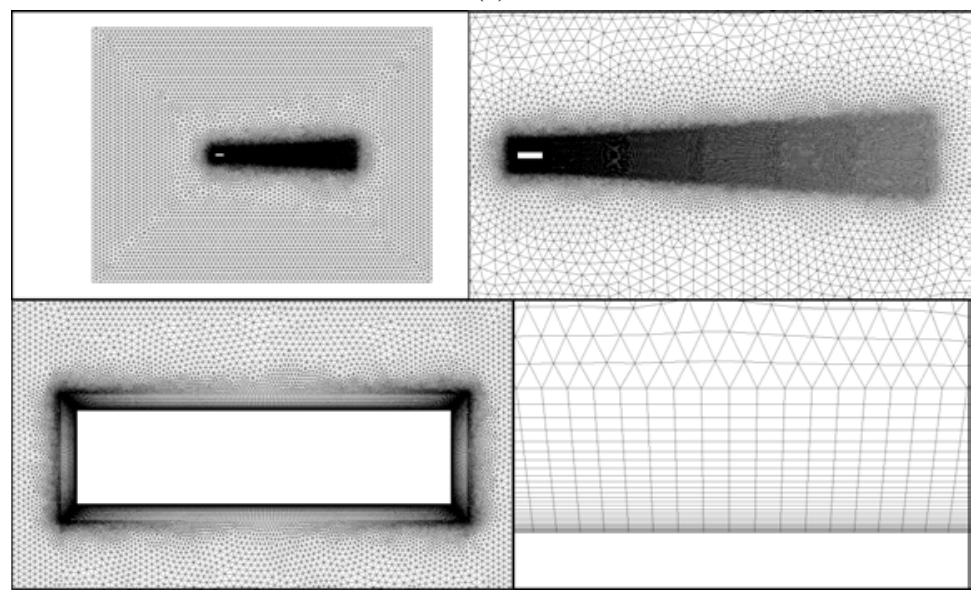

(b)

Figure 1: (a) Computational fluid domain; (b) Mesh characteristics (left top: overall mesh; right top: wake and wake buffer; left bottom: boundary layer; right bottom: boundary layer detail). 
was allowed to move in the heaving mode only, and its oscillation was modelled by a singledegree-of-freedom mass-spring-damper system. The coupling between the fluid, structure and dynamic mesh was achieved by the use of the conventional serial staggered scheme when using OpenFoam, while a second order trapezoidal scheme was used with STAR-CCM+.

The overall computational domain and its dimensions are shown in Fig. 1(a), which are very similar to the ones reported in Bruno et al. [10] for various 5:1 rectangular cylinder simulations. At the inlet, Dirichlet conditions were imposed for the velocity, the specific dissipation and turbulent kinetic energy, meanwhile Neumann conditions were imposed for the pressure. At the outlet, Dirichlet conditions were applied to the pressure, and Neumann conditions to the velocity, the specific dissipation and turbulent kinetic energy. For the Reynolds stresses in the RSTM simulations, Dirichlet conditions were applied in both the inlet and outlet. All the simulations were performed in low turbulence flow ( $I=1 \%, I$ is the turbulence intensity). For the rectangular cylinder walls, no-slip and no-penetration boundary conditions were applied [20].

\section{VERIFICATION STUDY AND STATIC CASE RESULTS}

The characteristics of the different meshes considered in the verification studies of this work are presented in Table 1, while images of the so-called medium mesh are shown in Fig. 1(b). The main difference between the different meshes is a finer discretisation of the $\mathbf{L}, \mathbf{M}$ and $\mathbf{N}$ zones from the coarse to the fine mesh (see Fig. 1(a)).

In this verification study, conducted considering the $k-\omega$ SST turbulence model, the values of the bulk parameters (force coefficients and Strouhal number) were the key elements to consider. Their values for the different meshes are presented in Table 2. It can be seen

Table 1: Mesh properties. The parameter $y_{1}$ is the total height of the first element of the boundary layer $(\mathrm{bl}), B$ is the width of the rectangular cylinder, $x_{1}$ is the length of first element in the bl, $r$ is the growth ratio of the elements in the bl, $n_{b l}$ is the number of layers forming the bl and $y_{b l}$ is the total height of the bl.

\begin{tabular}{lcccccccc}
\hline & & & & & & Quadrilateral & Triangular \\
Mesh & $y_{1} / B$ & $x_{1} / y_{1}$ & $r$ & $n_{b l}$ & $y_{b l} / B$ & cells & cells & Total cells \\
\hline Coarse & $1.97 \cdot 10^{-4}$ & 4 & 1.11 & 30 & $4.3264 \cdot 10^{-2}$ & 29,640 & 59,892 & 89,532 \\
Medium & $1.97 \cdot 10^{-4}$ & 4 & 1.11 & 30 & $4.3264 \cdot 10^{-2}$ & 29,640 & 105,088 & 134,728 \\
Fine & $1.97 \cdot 10^{-4}$ & 4 & 1.11 & 30 & $4.3264 \cdot 10^{-2}$ & 29,640 & 143,728 & 173,368 \\
\hline
\end{tabular}

Table 2: Bulk parameters for the static cases. (All data calculated respect D.)

\begin{tabular}{lcccccccc}
\hline Mesh & Method & $\operatorname{Re}$ & $I[\%]$ & $\overline{C_{d}}$ & $\widetilde{C_{d}}$ & $\widetilde{C_{l}}$ & $\widetilde{C_{m}}$ & $S t$ \\
\hline Coarse & $k-\omega S S T$ & $0.5 \mathrm{e} 5$ & 1 & 1.178 & 0.020 & 0.974 & 0.625 & 0.135 \\
Medium & $k-\omega S S T$ & $0.5 \mathrm{e} 5$ & 1 & 1.188 & 0.019 & 0.973 & 0.626 & 0.137 \\
Fine & $k-\omega S S T$ & $0.5 \mathrm{e} 5$ & 1 & 1.191 & 0.020 & 0.977 & 0.628 & 0.137 \\
\hline
\end{tabular}

Table 3: $y^{+}$values for the medium mesh.

\begin{tabular}{lcccc}
\hline Mesh & $\overline{y^{+}}$ & $y_{\max }^{+}$ & $\% y^{+}>2$ & $\% y^{+}>4$ \\
\hline Medium & 1.265 & 7.094 & 13.36 & 1.92 \\
\hline
\end{tabular}


Table 4: Validation of the static cases. (All data calculated respect D.)

\begin{tabular}{lcccccccc}
\hline & Method & $R e$ & $I[\%]$ & $\overline{C_{d}}$ & $\widetilde{C_{d}}$ & $\widetilde{C_{l}}$ & $\widetilde{C_{m}}$ & $S t$ \\
\hline Present study & $k-\omega S S T$ & $0.5 \mathrm{e} 5$ & 1.0 & 1.178 & 0.020 & 0.975 & 0.624 & 0.137 \\
Igarashi [22] & Exp. & $0.26-0.58 \cdot 10^{5}$ & - & 1.213 & - & - & - & 0.135 \\
Vairo [23] by CSTB & Exp. & $0.2 \cdot 10^{5}$ & - & 1.424 & 0.324 & 1.156 & 0.864 & 0.159 \\
Marra et al. [1] & Exp. & $0.15-0.57 \cdot 10^{5}$ & - & - & - & - & - & 0.136 \\
Sarkic [24] & Exp. & $0.38 \cdot 10^{5}-0.31 \cdot 10^{6}$ & $3.0-4.0$ & 0.924 & 0.14 & 0.544 & - & 0.129 \\
Sun et al. [25] & $k-\omega$ & $0.25 \mathrm{e} 5$ & 1.0 & 1.332 & 0.098 & 1.404 & 0.854 & 0.150 \\
\hline
\end{tabular}

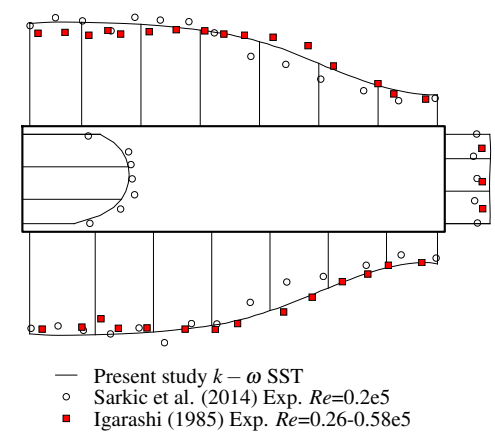

(a)

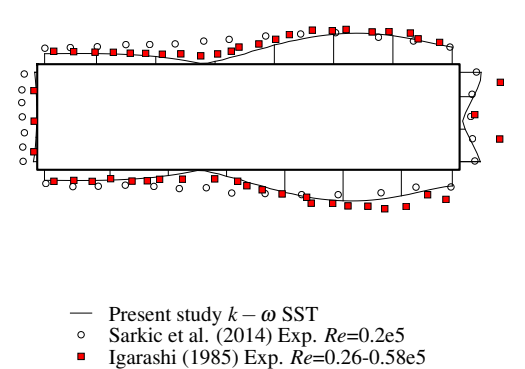

(b)

Figure 2: (a) Mean and $\left(\overline{C_{p}}\right)$; and (b) Standard deviation $\left(\widetilde{C_{p}}\right)$ pressure coefficient distributions. (Negative values points outwards for the $\overline{C_{p}}$ and the value of unity is equal to the cylinder height in both figures.)

that the discrepancies between the three meshes are very small. Thus, the medium mesh is retained for the rest of the simulations.

The values of the $y^{+}$were calculated considering the total height of the first element of the boundary layer. In all cases the mean value of $y^{+}$is $\overline{y^{+}} \leq 2$, obtaining for the medium mesh the values presented in Table 3 . The values obtained are very similar to the ones reported by Nieto et al. [21] for a rectangular cylinder of width to depth ratio 4.9:1.

In order to validate the results obtained from the CFD simulations adopting the $k-\omega$ SST turbulence model, the values of the bulk parameters and the mean $\left(\overline{C_{p}}\right)$ and fluctuating $\left(\widetilde{C_{p}}\right)$ pressure coefficients obtained for the medium mesh are compared with some available experimental and numerical data in Table 4 and Fig. 2 respectively. The results are in good agreement with the available data, and it is possible to identify the main flow characteristics from the pressure coefficient distributions, such as separation and reattachment.

Aiming to further study the main flow features in this aerodynamic problem, the time averaged streamlines and friction coefficient $\left(C_{f}\right)$ have been obtained, and they are shown in Fig. 3. The friction coefficient change in sign is linked with the different flow regions which develop over the cylinder surface. Consequently, for the mean flow the main vortices, covering almost the total length of the cylinder top and bottom faces, are identified. Towards the leeward edge, the flow reattaches and, finally, in the leeward edge a new vortex is formed. These regions are similar to the ones identified in Bruno et al. [26] for a 5:1 rectangular cylinder, adopting 3D LES simulation. The ratios of the cylinders studied, 4:1 and 5:1 


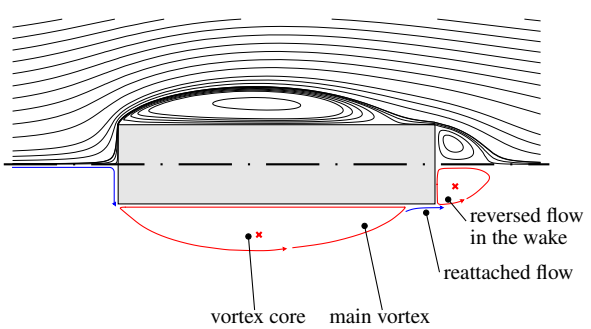

(a)

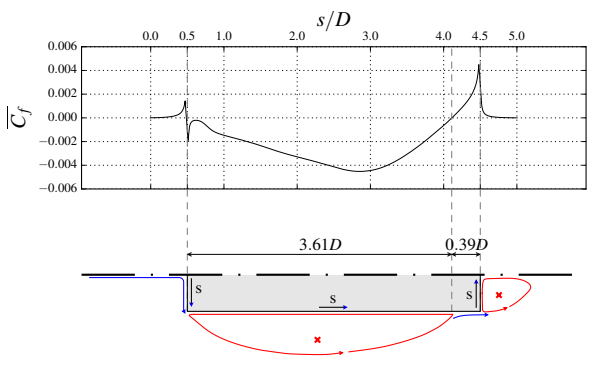

(b)

Figure 3: (a) Time averaged streamlines; (b) Time averaged, side averaged friction coefficient distribution.

respectively, are very close and thus, according to Bruno et al. [10], they show similar aerodynamic behaviour, characterised by a permanent reattached flow. In the present study no recirculation region close to the windward corner on the top and bottom faces of the cylinder is identified.

\section{RESULTS FOR THE DYNAMIC CASE}

The simulations for the vertically free-to-oscillate rectangular cylinder were performed for a Scruton number of $6.0(S c=(4 \pi M \xi) /(\rho B D L)$, where $M$ is the mass of the cylinder, $\xi$ is the relative damping, $\rho$ is the air density and $L$ is the spanwise dimension). The Scruton number is the same as in the experiments carried out by Marra et al. [1], which are adopted here for validation. The results obtained for the different simulations can be seen in Fig. 4. In this application case two different turbulence models, the $k-\omega$ SST and the RSTM, have been used in order to conduct the VIV simulations (see Fig. 4(a)). It can be seen, that both models overestimate the value of the peak amplitude. This overestimation is substantially reduced when the RSTM model is used, which is more complex than the $k-\omega \mathrm{SST}$, but at the expense of a greater computational cost, both in time and resources. This overestimation is mainly explained for the perfect correlation along the spanwise dimension of the simulations, due to the two dimensionality of the CFD models and consequently it is not possible to reproduce the three dimensionality of the flow over the cylinder. Moreover, the difference between the peak amplitudes obtained by the two turbulence models, can be explained as the $k-\omega$ SST models is an isotropic model, which does not resolve all the turbulent scales, concentrating them and yielding more energetic vortices, affecting the value of the aerodynamic damping. In order to account for the flow three dimensionality and resolution of different turbulent scales, LES simulations have been carried out in a different study, where it was found that the mesh discretisation, both in the spanwise direction and in the xy plane, plays a key role [27].

Both models are able to identify the sub-harmonic vortex-excitation region although in a wider range of reduced velocities in the case of the RSTM. Also, for both models, the fluctuation of the structural response and lift force are locked into the natural frequency of the model $\left(f_{0}\right)$ during the VIV lock-in (see Fig. 4(b)). The termination of the lock-in region coincides with the instance when the lift force and vertical displacement are out of phase (see Fig. 4(c)) [15]. The length of the lock-in region is overestimated in both cases. This can be explained by the delay of the point at which the $C_{l}$ and the oscillation are out of phase. Moreover, at the end of the lock-in region the vibration obtained by the $R S T M$ is not 


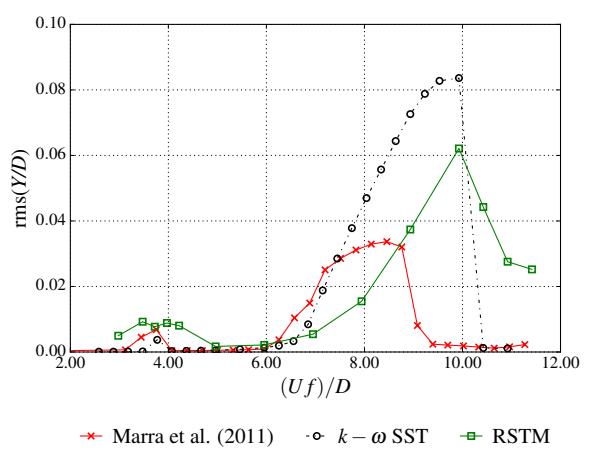

(a)

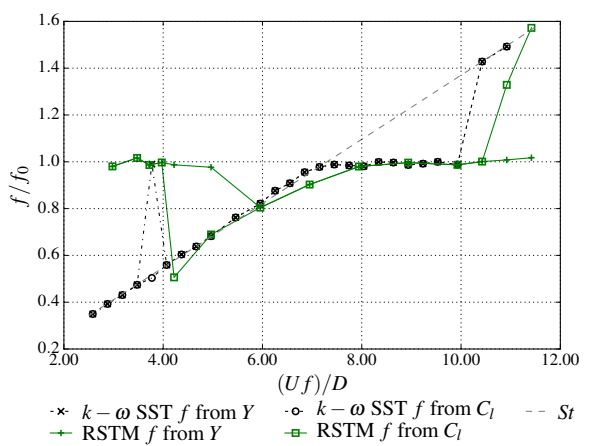

(b)

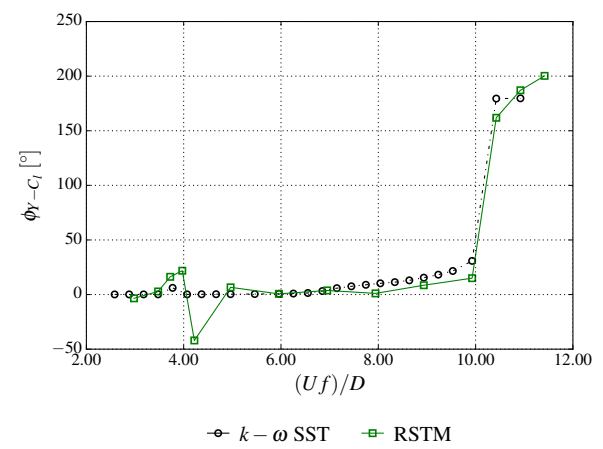

(c)

Figure 4: (a) Vibration amplitude; (b) Vibration frequency; (c) Phase lag between the vibration and the $C_{l}$.

of negligible. In all cases the cylinder was released from a location different to the balance position, what seems to be enough to obtain the sustained movement of the cylinder. Further studies must be conducted on this subject.

\section{CONCLUSIONS}

Numerical simulations for the 4:1 rectangular cylinder have been conducted using the $k-\omega$ SST turbulence model for the static and vertically free to oscillate configuration and the $R S T M$ for the latter one. The static simulations conducted with the $k-\omega$ SST turbulence model, present force coefficients, Strouhal number and pressure coefficient distributions in good agreement with the available experimental data, being able to identify different structures of the flow around the cylinder. The vertically free to oscillate simulations are able to identify the main VIV region as well as the sub-harmonic one, what makes this approach a feasible tool for the identification of this phenomenon. The peak value of the vibrations is overestimated, arguably due to the perfect spanwise correlation of the models, the incapacity of the models to reproduce the three dimensional flow features that takes places over the cylinder and the inherent limitations of the selected models. The use of more complex models reduces the value of the peak vibration value, but at the expense of increasing the computational cost of the simulations. In a different study, 3D LES simulation 
have been carried out, obtaining results closer to the experimental ones for the VIV case. Following this idea, 3D simulations using the RSTM model may be conducted, although the high computational burden associated to this turbulence model may penalise this approach.

\section{ACKNOWLEDGEMENTS}

This research has been funded by the Spanish Ministry of Economy and Competitiveness in the frame of the research project with reference BIA2016-76656-R and the Galician regional government with reference ED431C 2017/72. The first of the authors has been funded by the Spanish Ministry of Economy and Competitiveness in the frame of the National Program for Promotion of Talent and Employability through the BES-2014-068418 predoctoral contract grant, associated with the BIA2013-41965-P research project.

The authors fully acknowledge the received support.

\section{REFERENCES}

[1] Marra, A., Mannini, C. \& Bartoli, G., Van der Pol-type equation for modeling vortexinduced oscillations of bridge decks. Journal of Wind Engineering and Industrial Aerodynamics, 99, pp. 776-785, 2011.

[2] Nakaguchi, H., Hashimoto, K. \& Muto, S., An experimental study on aerodynamic drag of rectangular cylinder. Journal of the Japan Society of Aeronautical Engineering, 16(168), pp. 1-5, 1968.

[3] Mizota, T. \& Okajima, A., Experimental studies of unsteady flows around rectangular prisms. Proceedings of the Japan Society of Civil Engineers, 312, pp. 49-57, 1981.

[4] Okajima, A., Flow around a rectangular cylinder with a section of various width/height ratios. Journal of Wind Engineering, Japan Association of Wind Engineering, 17, pp. 119, 1983.

[5] Washizu, K., Ohya, A., Otsuki, Y. \& Fujii, K., Aeroelastic instability of rectangular cylinders in a heaving mode. Journal of Sound and Vibration, 59(2), pp. 195-210, 1978.

[6] Washizu, K., Ohya, A., Otsuki, Y. \& Fujii, K., Aeroelastic instability of rectangular cylinders in a torsional mode due to a transverse wind. Journal of Sound and Vibration, 72(4), pp. 507-521, 1980.

[7] Komatsu, S. \& Kobayashi, H., Vortex-induced oscillation of bluff cylinders. Journal of Wind Engineering and Industrial Aerodynamics, 6, pp. 335-362, 1980.

[8] Marra, A., Mannini, C. \& Bartoli, G., Measurements and improved model of vortexinduced vibration for an elongated rectangular cylinder. Journal of Wind Engineering and Industrial Aerodynamics, 147, pp. 358-367, 2015.

[9] Ohya, Y., Nakamura, Y., Ozono, S. \& Tsuruta, H., A numerical study of vortex shedding form flat plates with square leading and trailing edges. Journal of Fluids and Structures, 236, pp. 445-460, 1992.

[10] Bruno, L., Salvetti, M. \& Ricciardelli, F., Benchmark on the aerodynamics of a rectangular 5:1 cylinder: an overview after the first four years of activity. Journal of Wind Engineering and Industrial Aerodynamics, 126, pp. 87-106, 2014.

[11] Ricci, M., Patruno, L., de Miranda, S. \& Ubertini, F., Flow field around a 5:1 rectangular cylinder using LES: influence of inflow turbulence conditions, spanwise domain size and their interaction. Computers and Fluids, 149, pp. 181-193, 2017.

[12] Mannini, C., Soda, A. \& Schewe, G., Numerical investigation on the three-dimensional unsteady flow past a 5:1 rectangular cylinder. Journal of Wind Engineering and Industrial Aerodynamics, 99, pp. 469-482, 2011. 
[13] Shimada, K. \& Ishihara, T., Predictability of unsteady two-dimensional $k$ - $\epsilon$ model on the aerodynamic instabilities of some rectangular prisms. Journal of Fluids and Structures, 28, pp. 20-39, 2012.

[14] Daniels, S., Castro, I. \& Xie, Z., Numerical analysis of freestream turbulence effects on the vortex-induced vibrations of a rectangular cylinder. Journal of Wind Engineering and Industrial Aerodynamics, 153, pp. 13-25, 2015.

[15] Nguyen, D., Hargreaves, D. \& Owen, J., Vortex-induced vibration of a 5:1 rectangular cylinder: a comparison of wind tunnel sectional model tests and computational simulations. Journal of Wind Engineering and Industrial Aerodynamics, 175, pp. 116, 2018.

[16] Wilcox, D., Turbulence Modelling for CFD, La Cañada: DCW Industries, 3rd ed., 2006.

[17] Menter, F. \& Esch, T., Elements of industrial heat transfer prediction. Proceedings of the 16th Brazilian Congress of Mechanical Engineering, 20, pp. 117-127, 2001.

[18] Manceau, R. \& Hanjalić, K., Elliptic blending model: a new near-wall Reynolds-stress turbulence closure. Physics of Fluids, 14, pp. 744-754, 2002.

[19] Siemens automation STAR-CCM+ User Guide, 2016.

[20] Wilcox, D., Basic Fluid Mechanics, La Cañada: DCW Industries, 5th ed., 2012.

[21] Nieto, F., Owen, J., Hargreaves, D. \& Hernandez, S., Bridge deck flutter derivatives: efficient numerical evaluation exploiting their interdependence. Journal of Wind Engineering and Industrial Aerodynamics, 136, pp. 138-150, 2015.

[22] Igarashi, T., Characteristics of the flow around rectangular cylinders. Bulletin of the Japan Society of Mechanical Engineers, 28(242), pp. 1690-1696, 1985.

[23] Vairo, G., A numerical model for wind loads simulation on long-span bridges. Simulation Modelling Practice and Theory, 11, pp. 315-351, 2003.

[24] Sarkic, A., Validated numerical simulation of fluid structure interactions of bridge girders in turbulent wind fields. PhD thesis, Ruhr-Universität, Bochum, Germany, 2014.

[25] Sun, D., Owen, J. \& Wright, N., Application of the $k-\omega$ turbulence model for a windinduced vibration study of 2D bluff bodies. Journal of Wind Engineering and Industrial Aerodynamics, 97, pp. 77-87, 2009.

[26] Bruno, L., Fransos, D., Coste, N. \& Bosco, A., 3D flow around a rectangular cylinder: a computational study. Journal of Wind Engineering and Industrial Aerodynamics, 98, pp. 263-276, 2010.

[27] Álvarez, A., Nieto, F., Nguyen, D., Owen, J. \& Hernández, S., 3D LES simulations of a static and vertically free-to-oscillate 4:1 rectangular cylinder: effect of the spanwise grid resolution. Proceedings of 7th International Conference Symposium on Wind Engineering, Seoul, Republic of Korea, 2018. 